\title{
Erratum
}

\section{Monotone Decrease of Characteristic Functions}

\section{Richard S. Ellis ${ }^{1}$}

Received June 30, 1977

The hypotheses of the theorem in my paper ${ }^{(1)}$ are incorrectly stated. I thank Mark Pinsky for pointing this out to me.

Let $\rho$ denote the probability measure associated with the $n$-dimensional distribution function $F(x)$; i.e., for any Borel set $A \subseteq R^{n}$, we define

$$
\rho(A)=\int_{A} d F(x)
$$

Then hypotheses (1a) and (1b) should read as follows:

$$
\begin{gathered}
\rho \text { is symmetric } \\
\infty>\int(t \cdot x)^{2} d F(x)>0, \quad \text { all } t \in R^{n}, \quad t \neq 0
\end{gathered}
$$

Hypothesis (1a) implies that the characteristic function $f(t)=\int \exp (i t \cdot x) d F(x)$, $t \in R^{n}$, is real-valued. ${ }^{(2)}$ Also, in order to avoid any confusion, the last sentence in the theorem should read as follows: "Thus, in a suitable neighborhood of the origin, $f$ is monotonically decreasing along rays starting at the origin."

\section{REFERENCES}

1. R. S. Ellis, J. Stat. Phys. 16:117 (1977).

2. K. L. Chung, $A$ Course in Probability Theory (Harcourt, Brace, and World, New York, 1968), p. 145.

${ }^{1}$ Department of Mathematics and Statistics, University of Massachusetts, Amherst, Massachusetts. 